\title{
Potencial de Espécies Vegetais na Remediação de Solo Contaminado COM Sulfentrazone ${ }^{1}$
}

\author{
Potential of Plant Species in the Remediation of Soil Contaminated with Sulfentrazone
}

BELO, A.F. ${ }^{2}$, COELHO, A.T.C.P. ${ }^{3}$, FERREIRA, L.R. ${ }^{4}$, SILVA, A.A. ${ }^{4}$ e SANTOS, J.B. ${ }^{5}$

\begin{abstract}
RESUMO - Objetivou-se, neste trabalho, avaliar a eficiência de espécies vegetais na remediação de um Argissolo Vermelho-Amarelo contaminado com sulfentrazone. O trabalho foi conduzido em duas etapas. Na primeira, avaliou-se o crescimento de Helianthus annus, Canavalia ensiformis, Dolichos lab lab e Arachis hypogaea em solo contaminado com sulfentrazone; na segunda, cultivou-se, no mesmo solo, uma espécie (sorgo) indicadora de resíduo de sulfentrazone no solo, para avaliar a capacidade remediadora dessas espécies. Na primeira etapa, foram utilizados vasos contendo $6,0 \mathrm{~kg}$ do substrato. Após a irrigação dos vasos, aplicou-se na superficie do solo o herbicida. Um dia após essa aplicação, procedeu-se à semeadura das espécies vegetais, as quais foram colhidas 100 dias depois e secas em estufa, determinandose a matéria seca da parte aérea. $\mathrm{Na}$ segunda etapa, foram coletadas amostras de 3,0 kg de solo de cada vaso, onde foi cultivada a planta indicadora. Aos 20 e 50 dias após a emergência, foi avaliada, visualmente, a intoxicação das plantas de sorgo, sendo determinada a matéria seca da parte aérea dessas plantas aos 50 dias após a emergência e aos 50 dias após o primeiro corte. A produção de matéria seca da parte aérea de H. annus, C. ensiformis, D. lab lab e A. hypogaea não foi alterada, indicando que essas espécies foram tolerantes ao sulfentrazone; entretanto, $H$. annus apresentou melhor capacidade para remediação de solo contaminado com esse herbicida.
\end{abstract}

Palavras-chave: descontaminação de solo, fitorremediação, Helianthus annus, Canavalia ensiformis, Dolichos lab lab, Arachis hypogaea.

\begin{abstract}
The aim of this work was to evaluate the efficiency of plant species in the remediation of a red-yellow Hapludalf contaminated with sulfentrazone. The work was conducted at two stages. At the first, the growth of Helianthus annus, Canavalia ensiformis, Dolichos lab lab and Arachis hypogaea was evaluated in soil contaminated with sulfentrazone. At the second stage, a species (sorghum) indicator of residues of this herbicide was sown in the same soil. At the first stage, pots containing $6.0 \mathrm{~kg}$ of substrate were used. After the pots were irrigated, the herbicide was applied on the soil surface. One day after the application, the vegetal species were sown. These species were collected 100 days later and stove-dried for determination of shoot dry matter accumulation. At the second stage, soil samples of $3.0 \mathrm{~kg}$ were collected from each pot where the species indicator of sulfentrazone residues was cultivated. At 20 and 50 days after emergence, the intoxication of the sorghum plants was visually determined and the shoot dry matter of these plants was determined 50 days after emergence and 50 days after the first cut. The production of shoot dry matter of $\boldsymbol{H}$. annus, C. ensiformis, D. lab lab and $\boldsymbol{A}$. hypogaea was not affected, indicating that all species were tolerant to sulfentrazone. However, $\boldsymbol{H}$. annus showed greater capacity for remediation of soil contaminated with this herbicide.
\end{abstract}

Keywords: soil decontamination, phytoremediation, Helianthus annus, Canavalia ensiformis, Dolichos lab lab, Arachis hypogaea.

Recebido para publicação em 26.8.2010 e aprovado em 16.6.2011.

2 Engâ-Agra ${ }^{\mathrm{a}}$, M.Sc., Doutora em Fitotecnia, Dep. de Fitotecnia, Universidade Federal de Viçosa - DFT/UFV, Campus UFV, 36570-000 Viçosa-MG, <ferreiragro@yahoo.com.br>; ${ }^{3}$ Engo-Agro ${ }^{\circ}$, UFV; ${ }^{4}$ Engo-Agr ${ }^{\circ}$., D.Sc., Professor, DFT/UFV, Campus-UFV; ${ }^{5} \mathrm{Eng}^{\mathrm{0}}-\mathrm{Agr}^{0}$, D.Sc., Professor da Universidade Federal dos Vales do Jequitinhonha e Mucuri, Faculdade de Ciências Agrárias, Campus II - Rodovia MGT 367 - Km 583, n 5000, Bairro Alto da Jacuba, 39100-000 Diamantina-MG. 


\section{INTRODUÇÃO}

As atividades industriais, de mineração e de agricultura têm sido apontadas como as principais responsáveis pela contaminação do solo, cursos de água e lençol freático por diferentes compostos químicos. Atualmente, o aumento dos insumos químicos na agricultura - entre eles, os herbicidas - tem preocupado a sociedade em geral, em razão da contaminação ambiental.

O herbicida sulfentrazone - N-[2,4-dicloro5[4-(diflurometil)-4,5-di-hidro-3 metil-5-oxo-1 H-1,2,4-triazol-1-il] metanosulfonamida - é registrado no Brasil para o controle de plantas daninhas em pré-emergência nas culturas de cana-de-açúcar, soja, citros, café, eucalipto e em áreas não agrícolas. A absorção desse herbicida ocorre pelo sistema radicular e sua translocação se dá por pequena movimentação pelo floema. Ele age nas plantas por um processo de ruptura da membrana celular, provocando rápida dessecação foliar nas plantas que emergem (Rodrigues \& Almeida, 2005). É um herbicida inibidor da Protox (protoporfirinogênio oxidase), registrado para aplicação em pré ou pós-emergência das plantas daninhas, controlando amplo espectro de folhas largas e gramineas.

A dissipação do sulfentrazone está diretamente relacionada com a disponibilidade de água, que pode melhorar as condições de degradação da molécula ou mesmo lixiviá-la em profundidade no solo (Ohmes et al., 2000).

$\mathrm{O}$ efeito residual prolongado do sulfentrazone no solo pode acarretar danos às culturas sucedâneas suscetiveis, como milheto, aveia-preta e sorgo (Pereira et al., 2000). Além disso, pode também provocar intoxicação no milho em sucessão à soja (Artuzi \& Contiero, 2006) e em milheto, aveia e trigo; contudo, não prejudicou o desenvolvimento das culturas de girassol e feijão (Blanco \& Velini, 2005).

A persistência do sulfentrazone em Argissolo Vermelho-Amarelo cultivado com cana-de-açúcar foi de 640 e 197 dias após a aplicação em 2003 e 2004, respectivamente, indicando o alto risco de intoxicação das culturas em sucessão à cana-de-açúcar (Vivian et al., 2006). Reduções superiores a $30 \%$ na produção de algodão foram avaliadas por Main et al. (2004).

Quando a molécula de um herbicida chega ao solo, ela pode ser degradada por processos químicos e biológicos, ser sorvida aos coloides do solo ou lixiviada. A sorção e a degradação do herbicida irão definir a disponibilidade do produto na solução do solo para ser absorvido pelas plantas ou lixiviado para camadas subsuperficiais do solo, podendo atingir os cursos de água subterrâneos (Prata \& Lavorenti, 2000).

A biorremediação é o processo de remediação normalmente in situ de áreas contaminadas que emprega organismos vivos (microrganismos e plantas) capazes de se desenvolver em meio contendo o material poluente, reduzindo-o ou até mesmo eliminando sua toxicidade. Essa técnica é utilizada para remediação de áreas contaminadas com metais pesados (Franco, 2004; Querol et al., 2006) e herbicidas (Santos et al., 2007; Carmo et al., 2008). Quando se trata da descontaminação pela utilização de plantas isoladas ou estimulando a microbiota associada às suas raízes, tem-se a fitorremediação (Wilson et al., 2000).

A fitorremediação tem se mostrado técnica promissora para descontaminação de solos contaminados com vários herbicidas, como tebuthiuron (Pires et al., 2005a,b), trifloxysulfuron-sodium (Santos et al., 2007) e picloram (Carmo et al., 2008). Assim, objetivouse, neste trabalho, avaliar o potencial das espécies Helianthus annus, Canavalia ensiformis, Dolichos lab lab e Arachis hypogaea na remediação de solo contaminado com sulfentrazone.

\section{MATERIAL E MÉTODOS}

Este trabalho, composto por duas etapas, foi realizado em casa de vegetação. Na primeira, avaliou-se o crescimento de espécies vegetais com potencial de descontaminação de solo contaminado com sulfentrazone. $\mathrm{O}$ experimento constou de um fatorial $4 \times 3$, no delineamento inteiramente casualizado, com quatro repetições. O primeiro fator foi constituído pelo cultivo das espécies $H$. annus, C. ensiformis, D. lab lab e A. hypogaea, e o segundo, por três doses $\left(0,250\right.$ e $\left.500 \mathrm{~g} \mathrm{ha}^{-1}\right)$ de sulfentrazone, aplicadas em pré-emergência. 
Na segunda etapa, avaliou-se a capacidade remediadora dessas espécies, através do crescimento de uma espécie indicadora (sorgo) de resíduos no solo desse herbicida. O experimento constou de um fatorial $5 \times 3$, no delineamento inteiramente casualizado, com quatro repetições. O primeiro fator foi constituído pelo cultivo ou não das espécies $H$. annus, C. ensiformis, D. lab lab e A. hypogaea, e o segundo, por três doses $(0,250$ e $500 \mathrm{~g} \mathrm{ha}^{-1}$ ) de sulfentrazone, aplicadas em pré-emergência.

Como substrato para o crescimento das plantas, utilizaram-se amostras de solo classificado como Argissolo Vermelho-Amarelo, cujas características físicas e químicas estão apresentadas na Tabela 1. As amostras do solo em estudo foram coletadas na profundidade de 0-20 cm, em área sem histórico de aplicação de herbicidas. Elas foram passadas em peneira com malha de $4 \mathrm{~mm}$ e adubadas com superfosfato simples na proporção de $10,0 \mathrm{~g} \mathrm{~kg}^{-1}$ de solo.

Na primeira etapa, foram utilizados vasos revestidos com filme de polietileno, contendo $6,0 \mathrm{~kg}$ do substrato. Após a irrigação dos vasos, visando atingir uma umidade próxima a $80 \%$ da capacidade de campo, fez-se a aplicação do herbicida com um pulverizador de precisão, equipado com bicos TT110.02, espaçados de $0,5 \mathrm{~m}$, calibrado para aplicação de $100 \mathrm{~L} \mathrm{ha}^{-1}$ de calda herbicida.

A semeadura das espécies vegetais potencialmente remediadoras (H. annus, C. ensiformis, D. lab lab e A. hypogaea) foi realizada um dia após a aplicação do sulfentrazone. Sete dias após a emergência foi realizado o desbaste, deixando-se três plantas por vaso. Para o bom crescimento e desenvolvimento das plantas, foram feitas irrigações diárias, mantendo-se a umidade do solo próxima a $80 \%$ da capacidade de campo. O controle da umidade do solo foi realizado por meio de pesagens semanais dos vasos, repondo-se a água evapotranspirada.

Aos 30, 45 e 60 dias após a emergência (DAE), foi adicionado em cada vaso $0,3 \mathrm{~g}$ de ureia, visando ao melhor crescimento das plantas. A partir dos 60 DAE foram feitas adubações semanais de cobertura, com $100 \mathrm{~mL}$ por vaso de solução Ouro Verde ${ }^{\circledR}$ contendo $\left(\mathrm{g} \mathrm{L}^{-1}\right): \mathrm{N}(3,75), \mathrm{P}_{2} \mathrm{O}_{5}(3,75), \mathrm{K}_{2} \mathrm{O}(5,00), \mathrm{CaO}$ $(0,75), \mathrm{S}(1,00), \mathrm{MgO}(0,20), \mathrm{Cl}(0,10), \mathrm{Mn}$ $(0,075), \mathrm{Fe}(0,015), \mathrm{B}(0,012)$ e $\mathrm{Zn}(0,010)$.

Aos 100 DAE, fez-se a colheita da parte aérea das plantas, que foram secas em estufa de circulação forçada de ar $\left(70 \pm 1^{\circ} \mathrm{C}\right)$ até massa constante, determinando-se a matéria seca de cada espécie em função dos tratamentos.

Na segunda etapa, foram coletadas amostras de solo de cada vaso da etapa anterior. Essas amostras foram secas ao ar e peneiradas, para retirada de raízes e eventuais torrões. Em seguida, foram colocados $3,0 \mathrm{~kg}$ desse solo em vasos revestidos com filme de polietileno, onde foi semeada a espécie indicadora (Sorghum bicolor), previamente selecionada como de alta sensibilidade ao sulfentrazone. Após a emergência das plântulas de sorgo, realizou-se o desbaste, deixandose seis plantas por vaso. Essas plantas foram irrigadas diariamente, para manutenção da

Tabela 1 - Resultados das análises física e química das amostras do Argissolo Vermelho-Amarelo utilizado no experimento

\begin{tabular}{|c|c|c|c|c|c|c|c|c|c|c|}
\hline \multicolumn{11}{|c|}{ Análise física $\left(\mathrm{dag} \mathrm{kg}^{-1}\right)^{1^{\prime}}$} \\
\hline Argila & & Silte & \multicolumn{2}{|c|}{ Areia fina } & \multicolumn{2}{|c|}{ Areia grossa } & \multicolumn{4}{|c|}{ Classificação textural } \\
\hline 24 & & 20 & \multicolumn{2}{|c|}{31} & \multicolumn{2}{|c|}{25} & \multicolumn{4}{|c|}{ Franco-argiloarenosa } \\
\hline \multicolumn{11}{|c|}{ Análise química $^{1 /}$} \\
\hline $\mathrm{pH}$ & $P$ & $\mathrm{~K}^{+}$ & $\mathrm{H}+\mathrm{Al}$ & $\mathrm{Al}^{3+}$ & $\mathrm{Ca}^{2+}$ & $\mathrm{Mg}^{2+}$ & $\mathrm{CTC}_{\mathrm{pH} 7,0}$ & $\mathrm{~V}$ & $\mathrm{~m}$ & $\mathrm{MO}$ \\
\hline$\left(\mathrm{H}_{2} \mathrm{O}\right)$ & \multicolumn{2}{|c|}{$\left(\mathrm{mg} \mathrm{dm}^{-3}\right)$} & \multicolumn{5}{|c|}{$\left(\mathrm{cmol}_{\mathrm{c}} \mathrm{dm}^{-3}\right)$} & \multicolumn{2}{|c|}{$(\%)$} & $\left(\right.$ dag kg $\left.{ }^{-1}\right)$ \\
\hline 5,0 & 49,7 & 122 & 3,63 & 0,1 & 5,9 & 1,5 & 11,34 & 68 & 1 & 1,3 \\
\hline
\end{tabular}

1/ Análises realizadas no Laboratório de Análise de Solos Viçosa Ltda. 
umidade do solo próximo a $80 \%$ da capacidade de campo, e adubadas com $50 \mathrm{~mL}$ de solução de Ouro Verde ${ }^{\circledR}$, conforme especificado na etapa anterior.

Aos 20 e 50 DAE, foi avaliada, visualmente, a intoxicação das plantas de sorgo pelo sulfentrazone. Atribuíram-se notas de 0 (ausência de intoxicação) a 100 (morte da planta). Aos $50 \mathrm{DAE}$, num primeiro corte, foi determinada a matéria seca da parte aérea das plantas de sorgo, ao passo que a matéria seca da rebrota do sorgo foi avaliada aos 50 dias após o primeiro corte, coincidindo com a colheita final. Essa avaliação foi realizada aos 200 dias após a aplicação do sulfentrazone no solo, ou seja, 100 dias cultivando as espécies vegetais potencialmente remediadoras e mais 100 dias cultivando sorgo.

Todos os dados obtidos foram submetidos à análise de variância. Na comparação das médias entre os tipos de cultivo utilizou-se o teste de Tukey a 5\% de probabilidade e, para avaliar os efeitos de doses do sulfentrazone no crescimento e desenvolvimento das plantas remediadoras, a análise de regressão. Os modelos foram escolhidos com base na significância dos coeficientes de regressão, utilizando-se o teste t a $5 \%$ de probabilidade, no fenômeno biológico e no coeficiente de determinação $\left(\mathrm{r}^{2}=\mathrm{SQReg} / \mathrm{SQT}\right.$ rat $)$.

\section{RESULTADOS E DISCUSSÃO}

O acúmulo de matéria seca da parte aérea das plantas de Helianthus annus, Canavalia ensiformis, Dolichos lab lab e Arachis hypogaea não foi influenciado pela ação do sulfentrazone, independentemente das doses aplicadas (Tabela 2), comprovando a tolerância dessas espécies ao herbicida, que é uma das características altamente desejável para que uma espécie seja utilizada como fitorremediadora. Dessa forma, $H$. annus, C. ensiformis, $D$. lab lab e A. hypogaea apresentam potencial para fitorremediação de solos tratados com sulfentrazone.

Não foram observados sintomas de intoxicação, aos 20 e 50 dias após a emergência (DAE), nas plantas de sorgo quando estas foram semeadas no solo com $250 \mathrm{~g} \mathrm{ha}^{-1} \mathrm{de}$ sulfentrazone após o cultivo com $H$. annus. Verificou-se, também para essa espécie, menor intoxicação nas plantas de sorgo (55\%) aos 20 DAE no solo com $500 \mathrm{~g} \mathrm{ha}^{-1}$ do herbicida (Tabela 3). Esses resultados mostram melhor eficiência de $H$. annus em remediar o solo contaminado com sulfentrazone, o que pode ser devido à maior absorção e metabolização do herbicida por essa espécie. Já foi evidenciado que $H$. annus apresenta maior absorção de metais pesados quando comparado com outras espécies de plantas, como Brassica juncea e Phaseolus coccineus (Dushenkov et al., 1997).

Embora tenha ocorrido intoxicação nas plantas de sorgo nos tratamentos com $500 \mathrm{~g} \mathrm{ha}^{-1}$ de sulfentrazone com cultivo prévio de $H$. annus, D. lab lab, A. hypogaea e C. ensiformis, verificou-se, aos $50 \mathrm{DAE}$, que esses sintomas foram menores quando comparados aos do tratamento sem cultivo prévio (Tabela 3).

Tabela 2 - Matéria seca da parte aérea de espécies fitorremediadoras, cultivadas por 100 dias em solo tratado ou não com sulfentrazone, com as respectivas equações de regressão

\begin{tabular}{|l|c|c|c|c|}
\hline \multirow{2}{*}{ Espécie fitorremediadora } & \multicolumn{3}{|c|}{ Matéria seca $(\mathrm{g})$} & \multirow{2}{*}{ Equação de regressão } \\
\cline { 2 - 4 } & 0 & 250 & 500 & \\
\cline { 2 - 5 } & 73,00 & 65,00 & 65,00 & $Y=67,67$ \\
\hline Helianthus annus & 156,00 & 173,00 & 173,00 & $Y=167,33$ \\
\hline Canavalia ensiformis & 107,00 & 126,00 & 126,00 & $Y=119,67$ \\
\hline Dolichos lab lab & 50,00 & 61,00 & 61,00 & $Y=57,33$ \\
\hline Arachis hypogaea & & 15,58 & & $Y$ \\
\hline \multicolumn{1}{|c|}{ CV $(\%)$} & & & \\
\hline
\end{tabular}


Tabela 3 - Efeito residual do sulfentrazone, aos 100 dias após sua aplicação, em solo após o cultivo ou não de espécies fitorremediadoras, expresso em porcentagem de intoxicação de plantas de sorgo aos 20 e 50 dias após a emergência (DAE), com as respectivas equações de regressão e coeficientes de determinação

\begin{tabular}{|c|c|c|c|c|c|}
\hline \multirow{3}{*}{ Espécie fitorremediadora } & \multicolumn{3}{|c|}{ Intoxicação (\%) } & \multirow{3}{*}{ Equação de regressão } & \multirow{3}{*}{$r^{2}$} \\
\hline & \multicolumn{3}{|c|}{ Doses $\left(\mathrm{g} \mathrm{ha}^{-1}\right)$} & & \\
\hline & 0 & 250 & 500 & & \\
\hline & \multicolumn{5}{|c|}{$20 \mathrm{DAE}$} \\
\hline Helianthus annus & $0,00 \mathrm{~A}^{\underline{1 /}}$ & $0,00 \mathrm{~B}$ & $55,00 \mathrm{C}$ & $Y=0,088^{*} \mathrm{D}$ & 0,727 \\
\hline Canavalia ensiformis & $0,00 \mathrm{~A}$ & $5,00 \mathrm{~B}$ & $77,50 \mathrm{~A}$ & $Y=0,128 * \mathrm{D}$ & 0,776 \\
\hline Dolichos lab lab & $0,00 \mathrm{~A}$ & $18,75 \mathrm{~A}$ & $71,25 \mathrm{AB}$ & $Y=0,129 * \mathrm{D}$ & 0,907 \\
\hline Arachis hypogaea & $0,00 \mathrm{~A}$ & $2,50 \mathrm{~B}$ & $63,75 \mathrm{BC}$ & $Y=0,104 * \mathrm{D}$ & 0,748 \\
\hline Sem cultivo prévio $^{\underline{2}}$ & $0,00 \mathrm{~A}$ & $30,00 \mathrm{~A}$ & $82,50 \mathrm{~A}$ & $Y=0,156^{*} \mathrm{D}$ & 0,940 \\
\hline \multirow[t]{2}{*}{$\mathrm{CV}(\%)$} & \multicolumn{3}{|c|}{24,19} & & \\
\hline & \multicolumn{5}{|c|}{$50 \mathrm{DAE}$} \\
\hline Helianthus annus & $0,00 \mathrm{~A}$ & $0,00 \mathrm{~B}$ & $23,75 \mathrm{~B}$ & $Y=0,038 * \mathrm{D}$ & 0,717 \\
\hline Canavalia ensiformis & $0,00 \mathrm{~A}$ & $8,75 \mathrm{~A}$ & $23,75 \mathrm{~B}$ & $Y=0,045^{*} \mathrm{D}$ & 0,841 \\
\hline Dolichos lab lab & $0,00 \mathrm{~A}$ & $0,00 \mathrm{~B}$ & $21,25 \mathrm{~B}$ & $Y=0,034 * \mathrm{D}$ & 0,739 \\
\hline Arachis hypogaea & $0,00 \mathrm{~A}$ & $0,00 \mathrm{~B}$ & $26,25 \mathrm{~B}$ & $Y=0,042 * \mathrm{D}$ & 0,723 \\
\hline Sem cultivo prévio ${ }^{2 /}$ & $0,00 \mathrm{~A}$ & $12,50 \mathrm{~A}$ & $61,65 \mathrm{~A}$ & $Y=0,108 * \mathrm{D}$ & 0,836 \\
\hline $\mathrm{CV}(\%)$ & \multicolumn{3}{|c|}{38,57} & & \\
\hline
\end{tabular}

1/ Médias seguidas pelas mesmas letras, na coluna, não diferem entre si pelo teste de Tukey a 5\% de probabilidade. ${ }^{2 /}$ Solo que permaneceu no vaso sem cultivo até o plantio do sorgo. ${ }^{*}$ Significativo a $5 \%$ pelo teste $t$.

Com o aumento da concentração do sulfentrazone no solo, observou-se comportamento linear crescente na intoxicação das plantas de sorgo aos 20 e 50 DAE. Todavia, nos solos remediados pelas espécies $H$. annus, $D$. lab lab, A. hypogaea e C. ensiformis o crescimento e desenvolvimento das plantas de sorgo evidenciaram menor disponibilidade de sulfentrazone no solo, quando comparado com o solo sem cultivo (Tabela 3). Neste trabalho, os sintomas de intoxicação do sulfentrazone em plantas de sorgo foram: redução da altura das plantas, clorose e necrose das folhas.

Embora na literatura não tenham sido encontrados trabalhos específicos sobre fitorremediação de solos com resíduos do herbicida sulfentrazone, vários autores mostram a eficiência dessa técnica para outros herbicidas: tebuthiuron (Pires et al., 2005a,b), trifloxysulfuron-sodium (Santos et al., 2007) e picloram (Carmo et al., 2008).

Não foram observadas diferenças no acúmulo de matéria seca da parte aérea (MSPA) das plantas de sorgo nos tratamentos com aplicação de 0 e $250 \mathrm{~g} \mathrm{ha}^{-1}$ de sulfentrazone (Tabela 4). Isso indica que em doses menores pode ter havido algum tipo de imobilização ou degradação do herbicida. Sabe-se que os microrganismos podem responder de forma diferenciada a doses de um herbicida, variando, então, o tempo requerido para sua degradação.

Martinez (2010) verificou que o herbicida sulfentrazone pode ser degradado por populações de microrganismos do solo; entre estes encontra-se o gênero Penicillium sp. Os fungos do gênero Penicillium sp. associamse com a rizosfera de plantas cultivadas (Silva \& Cavalcanti, 1990); esses organismos saprofiticos encontram-se em maior concentração na região do solo com maior aporte de carbono facilmente metabolizável, como na rizosfera das plantas, onde há deposição dos exsudatos radiculares. Os exsudatos radiculares das plantas apresentam grande importância para a degradação de compostos orgânicos, pois proporcionam condições favoráveis ao aumento da população microbiana, as quais por sua vez atuam diretamente na degradação. 
Tabela 4 - Efeito residual do sulfentrazone no solo após o cultivo ou não de espécies fitorremediadoras, expresso em matéria seca da parte aérea de plantas de sorgo cultivadas aos 100 dias após aplicação do herbicida e colhidas 50 dias após a emergência, com as respectivas equações de regressão e coeficientes de determinação

\begin{tabular}{|c|c|c|c|c|c|}
\hline \multirow{3}{*}{ Espécie fitorremediadora } & \multicolumn{3}{|c|}{ Matéria seca $(\mathrm{g})$} & \multirow{3}{*}{ Equação de regressão } & \multirow{3}{*}{$r^{2}$} \\
\hline & \multicolumn{3}{|c|}{ Doses $\left(\mathrm{g} \mathrm{ha}^{-1}\right)$} & & \\
\hline & 0 & 250 & 500 & & \\
\hline Helianthus annus & $26,77 \mathrm{~A}^{1 / /}$ & $25,58 \mathrm{~A}$ & $21,81 \mathrm{~A}$ & $Y=27,2037-0,0099 * \mathrm{D}$ & 0,353 \\
\hline Canavalia ensiformis & $24,22 \mathrm{~A}$ & $21,06 \mathrm{~A}$ & $17,60 \mathrm{AB}$ & $Y=24,2746-0,0132 * \mathrm{D}$ & 0,549 \\
\hline Dolichos lab lab & $24,20 \mathrm{~A}$ & $22,66 \mathrm{~A}$ & $21,00 \mathrm{~A}$ & $Y=22,62$ & - \\
\hline Arachis hypogaea & $23,52 \mathrm{~A}$ & $21,38 \mathrm{~A}$ & $18,28 \mathrm{AB}$ & $Y=23,6817-0,0105^{*} \mathrm{D}$ & 0,437 \\
\hline Sem cultivo prévio" & $24,38 \mathrm{~A}$ & $24,44 \mathrm{~A}$ & $13,60 \mathrm{~B}$ & $Y=26,1996-0,0216^{*} \mathrm{D}$ & 0,541 \\
\hline $\mathrm{CV}(\%)$ & & 13,99 & & & \\
\hline
\end{tabular}

1/ Médias seguidas pelas mesmas letras, na coluna, não diferem entre si pelo teste de Tukey a $5 \%$ de probabilidade. ${ }^{2 /}$ Solo que permaneceu no vaso sem cultivo até o plantio do sorgo. ${ }^{*}$ Significativo a $5 \%$ pelo teste $\mathrm{t}$.

Na dose de $500 \mathrm{~g} \mathrm{ha}^{-1}$, não se observou diferença no acúmulo de MSPA das plantas de sorgo nos tratamentos com cultivo prévio das espécies com potencial de fitorremediação. Todavia, os tratamentos com cultivo prévio de $H$. annus e $D$. lab lab foram superiores à testemunha sem cultivo (Tabela 4), evidenciando a maior eficiência dessas espécies na fitorremediação do solo contaminado com até $500 \mathrm{~g} \mathrm{ha}^{-1} \mathrm{de}$ sulfentrazone. De alguma forma, $H$. annus e $D$. lab lab podem ter estimulado sua microbiota rizosférica, resultando numa degradação mais eficiente do sulfentrazone.

Pires et al. (2005b) avaliaram a atividade rizosférica de quatro espécies vegetais com potencial de fitorremediação de solo contaminado com tebuthiuron e concluíram que a maior evolução de $\mathrm{CO}_{2}$ no solo rizosférico tratado com tebuthiuron ocorreu no solo cultivado com Canavalia ensiformis, sendo esta espécie a mais promissora para a fitorremediação desse herbicida. A ação das plantas, influenciando a microbiota rizosférica e acelerando a degradação de compostos no solo, é conhecida como fitoestimulação, a qual se constitui em um dos principais mecanismos de fitorremediação de herbicidas no solo. Segundo Santos et al. (2007), a maior capacidade apresentada por Stizolobium aterrimum na descontaminação de solos com resíduos do trifloxysulfuron-sodium envolve também o processo de fitoestimulação.
Comparando as espécies em cultivo prévio, nas doses testadas, não foi observado efeito no acúmulo de MSPA das plantas de sorgo após o cultivo de $D$. lab lab. Nos demais tratamentos foi verificada a redução da MSPA das plantas de sorgo com o aumento da concentração do sulfentrazone no solo (Tabela 4), o que confirma a diferença na capacidade remediadora das espécies vegetais testadas. Pires et al. (2005a), avaliando a capacidade de sete espécies vegetais na descontaminação de solos contaminados com tebuthiuron, observaram que até a dose de $0,5 \mathrm{~kg} \mathrm{ha}^{-1}$ do herbicida a espécie que melhor fitorremediou o solo foi Lupinus albus. No entanto, quando o solo foi tratado com $1,0 \mathrm{~kg} \mathrm{ha}^{-1}$ de tebuthiuron, Canavalia ensiformes foi a espécie que melhor fitorremediou o solo.

Não houve efeito do sulfentrazone sobre a MSPA da rebrota das plantas de sorgo nos tratamentos com cultivo prévio das espécies potencialmente fitorremediadoras. Somente se observou diminuição no acúmulo de MSPA dessas plantas no tratamento sem cultivo na dose de $500 \mathrm{~g} \mathrm{ha}^{-1}$, o que mais uma vez confirma a ação fitorremediadora das espécies avaliadas. Esses resultados também comprovam a longa persistência desse herbicida no solo na ausência das espécies fitorremediadoras, uma vez que até 200 dias após a aplicação o sulfentrazone ainda causa problemas às plantas de sorgo (Tabela 5), estando de acordo com Rodrigues \& Almeida (2005), que relataram meia-vida de 180 dias do sulfentrazone em solos brasileiros. 
Tabela 5 - Efeito residual do sulfentrazone no solo após o cultivo ou não de espécies fitorremediadoras, expresso em matéria seca da parte aérea da rebrota de plantas de sorgo cultivadas aos 150 dias após aplicação do herbicida e colhidas 50 dias após a emergência, com as respectivas equações de regressão e coeficientes de determinação

\begin{tabular}{|c|c|c|c|c|c|}
\hline \multirow{3}{*}{ Espécie fitorremediadora } & \multicolumn{3}{|c|}{ Matéria seca $(\mathrm{g})$} & \multirow{3}{*}{ Equação de regressão } & \multirow{3}{*}{$r^{2}$} \\
\hline & \multicolumn{3}{|c|}{ Doses $\left(\mathrm{g} \mathrm{ha}^{-1}\right)$} & & \\
\hline & 0 & 250 & 500 & & \\
\hline Helianthus annus & $7,66 \mathrm{~A}^{\mathrm{1}^{\prime}}$ & $5,38 \mathrm{~A}$ & $5,54 \mathrm{~A}$ & $\hat{\mathrm{Y}}=6,19$ & - \\
\hline Canavalia ensiformis & $7,48 \mathrm{~A}$ & $6,85 \mathrm{~A}$ & $6,75 \mathrm{~A}$ & $\hat{\mathrm{Y}}=7,03$ & - \\
\hline Dolichos lab lab & $6,60 \mathrm{~A}$ & $5,89 \mathrm{~A}$ & $6,04 \mathrm{~A}$ & $\hat{\mathrm{Y}}=6,18$ & - \\
\hline Arachis hypogaea & $5,81 \mathrm{~A}$ & $5,24 \mathrm{~A}$ & $5,18 \mathrm{~A}$ & $\hat{\mathrm{Y}}=5,41$ & - \\
\hline Sem cultivo prévio ${ }^{2 /}$ & $8,14 \mathrm{~A}$ & $7,68 \mathrm{~A}$ & $4,62 \mathrm{~B}$ & $\hat{\mathrm{Y}}=8,5767-0,0070 * \mathrm{D}$ & 0,359 \\
\hline $\mathrm{CV}(\%)$ & \multicolumn{3}{|c|}{31,92} & & \\
\hline
\end{tabular}

${ }^{1 /}$ Médias seguidas pelas mesmas letras, na coluna, não diferem entre si pelo teste de Tukey a $5 \%$ de probabilidade. ${ }^{2 /}$ Solo que permaneceu no vaso sem cultivo até o plantio do sorgo. ${ }^{*}$ Significativo a $5 \%$ pelo teste $t$.

Avaliando os tipos de cultivo, nas doses testadas, não se observaram diferenças no acúmulo de MSPA da rebrota de plantas de sorgo após o cultivo prévio de $H$. annus, C. ensiformis, D. lab lab e A. hypogaea. Todavia, no tratamento sem cultivo prévio, com o aumento da concentração do sulfentrazone no solo, constatou-se redução da matéria seca da parte aérea (Tabela 5).

Todas as espécies cultivadas são tolerantes ao sulfentrazone e apresentam potencial de remediação de solo contaminado com esse herbicida; entretanto, Helianthus annus apresenta melhor capacidade de remediação de solo contaminado com esse herbicida.

\section{AGRADECIMENTO}

Ao Conselho Nacional de Desenvolvimento Científico e Tecnológico - CNPq, pelo apoio financeiro para a realização deste trabalho.

\section{LITERATURA CITADA}

ARTUZI, J. P.; CONTIERO, R. L. Herbicidas aplicados na soja e produtividade do milho em sucessão. Pesq. Agropec. Bras., v. 41, n. 7, p. 1119-1123, 2006.

BLANCO, F. M. G.; VELINI, E. D. Persistência do herbicida sulfentrazone em solo cultivado com soja e seu efeito em culturas sucedâneas. Planta Daninha, v. 23, n. 4, p. 693-700, 2005.

CARMO, M. L. et al. Influência do período de cultivo de Panicum maximum (cultivar Tanzânia) na fitorremediação de solo contaminado com picloram. Planta Daninha, v. 26, n. 2, p. $315-322,2008$.
DUSHENKOV, S. et al. Removal of uranium from water using terrestrial plants. Environ. Sci. Technol., v. 31, n. 12, p. $3468-3474,1997$

FRANCO, L. O.; MAIA, R. C. C.; PORTO, A. L. F. Remoção de metais pesados por quitina e quitosana isoladas de Cunninghamella elegans. Braz. J. Microbiol., v. 35, n. 3, p. 243-247, 2004.

MAIN, C. L. et al. Sulfentrazone persistence in southern soils: bioavailable concentration and effect on a rotational cotton crop. Weed Technol., v. 18, n. 2, p. 346-352, 2004.

MARTINEZ, C. O. et al. Microbial degradation of sulfentrazone in a Brazilian Rhodic Hapludox soil. Braz. J. Microbiol., v. 41, n. 1, p. 209-217, 2010.

OHMES, G. A. et al. Sulfentrazone dissipation in a Tennessee soil. Weed Technol., v. 14, n. 1, p. 100-105, 2000.

PEREIRA, F. A. R. et al. Seletividade de sulfentrazone em cultivares de soja e efeitos residuais sobre culturas sucessivas em solos de cerrado. R. Bras. Herbic., v. 1, n. 3, p. 219-224, 2000 .

PIRES, F. R. et al. Fitorremediação de solos contaminados com tebuthiuron utilizando-se espécies cultivadas para adubação verde. Planta Daninha, v. 23, n. 4, p. 711-717, 2005a.

PIRES, F. R. et al. Inferências sobre atividade rizosférica de espécies com potencial para fitorremediação do herbicida tebuthiuron. R. Bras. Ci. Solo, v. 29, n. 4, p. 627-634, 2005 b.

PRATA, F.; LAVORENTI, A. Comportamento de herbicidas no solo: influência da matéria orgânica. R. Bioci., v. 6, n. 2, p. $17-22,2000$. 
QUEROL, X. et al. Immobilization of heavy metals in polluted soils by the addition of zeolitic material synthesized from coal fly ash. Chemosphere, v. 62, n. 2, p. 171-180, 2006.

RODRIGUES, B. N.; ALMEIDA, F. S. Guia de herbicidas. 5.ed. Londrina: Edição dos Autores, 2005. 591 p.

SANTOS, E. A. et al. Fitoestimulação por Stizolobium aterrimum como processo de remediação de solo contaminado com trifloxysulfuron-sodium. Planta Daninha, v. 25, n. 2 , p. $259-265,2007$.
SILVA, M. I. L.; CAVALCANTI, M. A. Q. Fungos na rizosfera de sementes de tomate (Lycopersicon esculentum). Fitopatol. Bras., v. 15, n. 4, p. 323-326, 1990.

VIVIAN, R. et al. Persistência de sulfentrazone em Argissolo Vermelho-Amarelo cultivado com cana-de-açúcar.

Planta Daninha, v. 24, n. 4, p. 741-750, 2006.

WILSON, P. C. et al. Phytotoxicity, uptake, and distribution of ${ }^{14} \mathrm{C}$-simazine in Acorus gramenius and Pontederia cordata. Weed Sci., v. 48, n. 6, p. 701-709, 2000. 